\title{
Analytical Expressions for Frequency and Buckling of Large Amplitude Vibration of Multilayered Composite Beams
}

\author{
R. A. Jafari-Talookolaei, M. H. Kargarnovin, M. T. Ahmadian, and M. Abedi \\ School of Mechanical Engineering, Sharif University of Technology, P.O. Box 14588-89694, Tehran, Iran \\ Correspondence should be addressed to M. H. Kargarnovin, mhkargar@sharif.edu
}

Received 9 May 2011; Accepted 24 May 2011

Academic Editor: K. M. Liew

Copyright (๑) 2011 R. A. Jafari-Talookolaei et al. This is an open access article distributed under the Creative Commons Attribution License, which permits unrestricted use, distribution, and reproduction in any medium, provided the original work is properly cited.

\begin{abstract}
The aim of this paper is to present analytical and exact expressions for the frequency and buckling of large amplitude vibration of the symmetrical laminated composite beam (LCB) with simple and clamped end conditions. The equations of motion are derived by using Hamilton's principle. The influences of axial force, Poisson effect, shear deformation, and rotary inertia are taken into account in the formulation. First, the geometric nonlinearity based on the von Karman's assumptions is incorporated in the formulation while retaining the linear behavior for the material. Then, the displacement fields used for the analysis are coupled using the equilibrium equations of the composite beam. Substituting this coupled displacement fields in the potential and kinetic energies and using harmonic balance method, we obtain the ordinary differential equation in time domain. Finally, applying first order of homotopy analysis method (HAM), we get the closed form solutions for the natural frequency and deflection of the LCB. A detailed numerical study is carried out to highlight the influences of amplitude of vibration, shear deformation and rotary inertia, slenderness ratios, and layup in the case of laminates on the natural frequency and buckling load.
\end{abstract}

\section{Introduction}

The uses of composite materials in structural components are increasing due to their attractive properties such as high strength/stiffness, light weight, and facility to materialize fiber orientation, material and stacking sequence. Beam-like structures have numerous applications in the industries such as aerospace and robotics. The increased use of the LCB requires a better understanding of large amplitude vibration aspects of them.

Nonlinear dynamic analysis of the LCB on the basis of classical lamination theory (CLT) has received a good amount of attention in the literature [1-6] while relatively little investigations $[7,8]$ have been performed for dynamic investigation of such beams with low slenderness ratio (length of the beam to the radius of gyration of the crosssection i.e. $=L / r)$ which have to be taken into account the shear deformation and rotary inertia. It should be noted that for the later, the equation of motion is complex, and even obtaining an approximate solution is much more difficult.
In our previous work in [1], we presented analytical expressions for large amplitude-free vibration and postbuckling analysis of unsymmetrically LCB on elastic foundation by using variational iteration method. We used the CLT to obtain differential equations of motion. A differential quadrature approach for nonlinear free vibration analysis of symmetric angle-ply laminated thin beams on nonlinear elastic foundation with elastically restrained against rotation edges has been studied by Malekzadeh and Vosoughi [2] Patel et al. [3] have extended a new three-nodded shear flexible beam element based on consistency approach to analyze the nonlinear flexural vibration and postbuckling behavior of simply supported laminated orthotropic composite beams on a two-parameter elastic foundation. The axial and transverse deformations of a geometrically nonlinear composite beam were considered to obtain a closed form solution for the postbuckling behavior of composite beams and to exactly solve for the free vibrations that take place around a buckled position by Emam and Nayfeh [4]. Kapania and Raciti [5] presented a two-nodded Timoshenko beam element with 10 degrees of freedom per 
node to examine the large amplitude free vibrations of LCB. The nonlinear free flexural vibrations of isotropic/laminated orthotropic straight/curved beams have been studied using a cubic B-spline shear flexible curved element [6]. The nonlinear governing equations are solved by employing Newmark's numerical integration scheme coupled with modified Newton-Raphson iteration technique. Kiran et al. [7] had investigated the large amplitude free vibrations of generally layered laminated composite beams by developing a finite element model. A one-dimensional finite element formulation based on a higher-order displacement model has been developed by Obst and Kapania [8]. The model accounts for geometric nonlinearities, a parabolic shear strain distribution through the thickness, and satisfies the shear stress free boundary conditions at the upper and lower surfaces of the beam. The model has been applied to the nonlinear static and transient analysis, free vibration analysis, and impact analysis of laminated beams.

To the best author's knowledge, there is no analytical expressions in the literature on the large amplitude free vibrations analysis of the symmetrically LCB on the basis of first shear deformation theory (FSDT). Thus, the contribution of this paper is to expand our previous work in [1] by studying the more complicated and important problem to obtain the closed-form expressions for natural frequency and deflection.

\section{Equations of Motion and Boundary Condition}

The considered LCB with simple end conditions is shown in Figure 1 with the coordinates $\hat{x}$ along the axis of the beam and $\hat{z}$ along the thickness direction. $L, P, \widehat{w}(\hat{x}, \hat{t}), \hat{t}$ are length, compressive axial load, deflection of the beam, and time, respectively.

2.1. Coupling Equations. Based on FSDT, the LCB is characterized by its bending rigidity EI, torsional rigidity GJ, bending-torsion coupling rigidity $\mathrm{C}$, and shear rigidity $\mathrm{kAG}$ [9]. The derivation of these rigidities is briefly summarized in Appendix A [10].

The kinetic and potential energies of the LCB and the work done by the static lateral load per unit length of the beam $F_{\mathrm{z}}$ can be written as

$$
\begin{gathered}
T=\frac{1}{2} \int_{0}^{L}\left[I_{1} \hat{w}_{\hat{,}}^{2}+I_{2} \theta_{, \hat{t}}^{2}+I_{p} \psi_{, \hat{t}}^{2}\right] d \hat{x} \\
U=\frac{1}{2} \int_{0}^{L}\left[\operatorname{EI} \theta_{, \hat{x}}^{2}-P\left(\widehat{w}_{, \hat{x}}^{2}+\frac{I_{p}}{I_{1}} \psi_{, \hat{x}}^{2}\right)+2 C \psi_{, \hat{x}} \theta_{, \hat{x}}\right. \\
\left.+\operatorname{kAG}\left(\widehat{w}_{, \hat{x}}-\theta\right)^{2}+\mathrm{GJ} \psi_{, \hat{x}}^{2}\right] d \hat{x} \\
W=\int_{0}^{L} F_{z} \hat{w} d \hat{x}
\end{gathered}
$$

where comma denotes derivative with respect to $x ; \theta, \psi$, are the angle of rotation and torsional rotation, respectively; $I_{1}, I_{2}$ and $I_{\mathrm{p}}$ are the first and second moment of inertia about

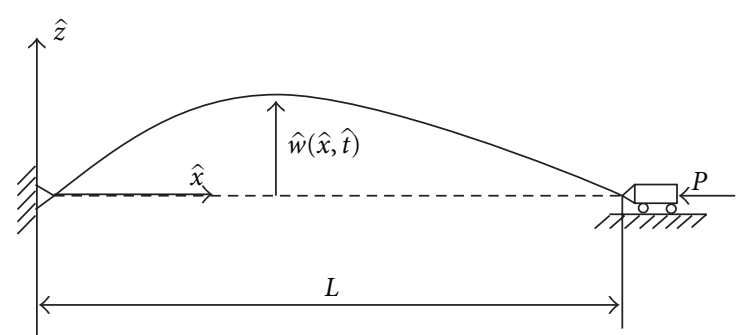

FIgURE 1: Geometry of a laminated composite beam.

$y$-axis and the polar mass moment of inertia per unit length about the $x$-axis, respectively.

For the subsequent results to be general, we use the following nondimensional variables:

$$
x=\frac{\hat{x}}{L}, \quad w=\frac{\hat{w}}{r}, \quad t=\frac{\hat{t}}{\sqrt{b D_{11} /\left(I_{1} L^{4}\right)}}, \quad r=\sqrt{\frac{I}{A}}
$$

which $r, b, I$, and $A$ are the radius of gyration of the cross-section, beam width, second moment of area, and the area of the cross-section, respectively, and $D_{11}$ is defined in Appendix A.

Thus, the kinetic and potential energy and the work done can be rewritten as follows:

$$
\begin{gathered}
T=\frac{I_{1} L^{5}}{2 b D_{11}} \int_{0}^{1}\left[I_{1} r^{2} w_{, t}^{2}+I_{2} \theta_{, t}^{2}+I_{p} \psi_{, t}^{2}\right] d x \\
U=\frac{1}{2} \int_{0}^{1}\left[\frac{\mathrm{EI}}{L} \theta_{, x}{ }^{2}-\frac{P}{L}\left(r^{2} w_{, x}{ }^{2}+\frac{I_{p}}{I_{1}} \psi_{, x}{ }^{2}\right)+\frac{2 C}{L} \psi_{, x} \theta_{, x}\right. \\
\left.+\frac{\mathrm{kAG}}{L}\left(r w_{, x}-L \theta\right)^{2}+\frac{\mathrm{GJ}}{L} \psi_{, x}{ }^{2}\right] d x \\
W=\int_{0}^{1} F_{z} w d x
\end{gathered}
$$

Applying the principle of minimization of total potential energy $(\delta(U-W))$, we obtain the following equilibrium equations as follows:

$$
\begin{gathered}
\frac{\mathrm{EI}}{L^{2}} \theta^{\prime \prime}+\mathrm{kAG}\left(\frac{r}{L} w^{\prime}-\theta\right)+\frac{\mathrm{C}}{L^{2}} \psi^{\prime \prime}=0, \\
\mathrm{kAG}\left(\frac{r}{L^{2}}-\frac{1}{L} \theta^{\prime}\right)-\frac{P r}{L^{2}} w^{\prime \prime}+F_{z}=0, \\
\frac{\mathrm{GJ}}{L^{2}} \psi^{\prime \prime}+\frac{\mathrm{C}}{L^{2}} \theta^{\prime \prime}-\frac{P I_{P}}{I_{1} L^{2}} \psi^{\prime \prime}=0
\end{gathered}
$$

in which prime denotes derivative with respect to nondimensional coordinate $x$. These equations are coupled equations and can be solved to get the coupled displacement fields [11].

2.2. Coupled Displacement Fields. Equations (4) and (6) are used to couple the rotation $\theta$, torsion $\psi$, and deflection of the beam $w$ [11]. These equations can be rewritten as

$$
\psi^{\prime \prime}=-A_{1} \theta^{\prime \prime}, \quad \theta^{\prime \prime}+A_{2} \theta=\frac{A_{2}}{\eta} w^{\prime},
$$


where

$$
A_{1}=\frac{C}{\mathrm{GJ}-\left(P I_{P} / I_{1}\right)}, \quad A_{2}=\frac{\mathrm{kAGL}}{C A_{1}-\mathrm{EI}},
$$

and $\eta$ is the slenderness ratio, that is, $\eta=L / r$.

2.2.1. Simple End Conditions. The boundary conditions for the simple end conditions beam are as follows:

$$
\begin{aligned}
(w= & 0, M=0, T=0)_{\text {at } x=0,1} \\
& \Longrightarrow\left(w=0, \theta^{\prime}=0, \psi^{\prime}=0\right)_{\text {at } x=0,1} .
\end{aligned}
$$

The transverse displacement $w$ is assumed as

$$
w(x, t)=u(t) \sin (\pi x),
$$

where $u(t)$ is the amplitude parameter and only is a function of $t$. Substituting the function $w$ in (7), we obtain the coupled rotation $\theta$ and torsion $\psi$ as

$$
\theta(x, t)=D_{1} u(t) \cos (\pi x), \quad \psi=-A_{1} D_{1} u(t) \cos (\pi x),
$$

where

$$
D_{1}=\frac{\pi A_{2}}{\eta\left(A_{2}-\pi^{2}\right)}
$$

2.2.2. Clamped End Conditions. In the case of the clampedclamped beam, the boundary conditions are as follows:

$$
(w=0, \theta=0, \psi=0)_{\text {at } x=0,1} .
$$

The admissible function for the $w$ is taken as follows:

$$
w=u(t)(1-\cos (2 \pi x)) .
$$

From (7) and (14), the coupled displacement fields for $\theta$ and $\psi$ are obtained as follows:

$$
\begin{gathered}
\theta=D_{2} u(t) \sin (2 \pi x), \\
\psi=-A_{1} D_{2} u(t) \sin (2 \pi x),
\end{gathered}
$$

where:

$$
D_{2}=\frac{2 \pi A_{2}}{\eta\left(A_{2}-4 \pi^{2}\right)} .
$$

2.3. Large Amplitude Vibrations. Consider that there is no external load on the beam, that is, $F_{z}=0$. The total energy for free oscillation of a system without damping is constant (energy conservation principle):

$$
T+U+W_{1}=\text { Constant, }
$$

where $W_{1}$ is the work done by the tension developed in the LCB due to the large amplitudes and is given by $[11,12]$

$$
W_{1}=\frac{E I r^{2}}{8 L^{3}}\left(\int_{0}^{1} w_{, x}^{2} d x\right)^{2}
$$

Substituting the expressions for $w, \theta$, and $\psi$, the expressions for $U, T$, and $W_{1}$ can be obtained as

$$
T=C_{1} \dot{u}^{2}, \quad U=\left(C_{2}-C_{3} P\right) u^{2}, \quad W_{1}=C_{4} u^{4}
$$

in which coefficients $C_{i}(i=1-4)$ for simple and clamped end conditions are expressed as simple end conditions:

$$
C_{1}=\frac{I_{1} L^{5}\left(I_{1} r^{2}+I_{P} A_{1}^{2} D_{1}^{2}+I_{2} D_{1}^{2}\right)}{4 b D_{11}},
$$

$C_{2}$

$$
\begin{aligned}
& =\frac{\pi^{2}\left(\mathrm{EI}_{1}{ }^{2}-2 C D_{1}{ }^{2} A_{1}\right)}{4 L} \\
& +\frac{\left(\mathrm{kAGL} L^{2} D_{1}{ }^{2}-2 \mathrm{kAG} r \pi L D_{1}+\mathrm{kAGr} r^{2} \pi^{2}+\mathrm{GJ} A_{1}{ }^{2} D_{1}{ }^{2} \pi^{2}\right)}{4 L},
\end{aligned}
$$

$C_{3}=\frac{\pi^{2}\left(I_{P} A_{1}^{2} D_{1}^{2}+r^{2} I_{1}\right)}{4 L I_{1}}$,

$$
C_{4}=\frac{\mathrm{EI} r^{2} \pi^{4}}{32 L^{3}}
$$

clamped end conditions:

$C_{1}=\frac{I_{1} L^{5}\left(3 I_{1} r^{2}+I_{P} A_{1}^{2} D_{2}^{2}+I_{2} D_{2}^{2}\right)}{4 b D_{11}}$,

$C_{2}$

$$
\begin{aligned}
& =\frac{\pi^{2}\left(\mathrm{EID}_{2}^{2}-2 \mathrm{CD}_{2}^{2} A_{1}\right)}{L} \\
& +\frac{\left(\mathrm{kAGL}{ }^{2} D_{2}^{2}-4 \mathrm{kAG} r \pi L D_{2}+4 \mathrm{kAGr} r^{2} \pi^{2}+4 \mathrm{GJ} A_{1}{ }^{2} D_{2}{ }^{2} \pi^{2}\right)}{4 L}, \\
& C_{3}=\frac{\pi^{2}\left(I_{P} A_{1}^{2} D_{2}^{2}+r^{2} I_{1}\right)}{L I_{1}} \\
& C_{4}=\frac{\mathrm{EI} r^{2} \pi^{4}}{2 L^{3}} .
\end{aligned}
$$

Substituting the expressions for $T, U$, and $W_{1}$ in (17), we get the following equation:

$$
C_{1} \dot{u}^{2}+\left(C_{2}-C_{3} P\right) u^{2}+C_{4} u^{4}=\text { Constant. }
$$

Differentiating (22), we obtain the nonlinear ordinary differential equations as follows:

$$
\ddot{u}+\frac{C_{2}-C_{3} P}{C_{1}} u+\frac{2 C_{4}}{C_{1}} u^{3}=0 \equiv \ddot{u}+\alpha u+\beta u^{3}=0 .
$$


In order to obtain the postbuckling load-deflection relation, one can set all time-derivative terms in (23) equal to zero which yields

$$
P_{\mathrm{NL}}=\frac{2 C_{4} u^{2}+C_{2}}{C_{3}} .
$$

Neglecting the contribution of $u$ in (24), the critical buckling load can be determined as

$$
P_{\mathrm{cr}}=\frac{C_{2}}{C_{3}} .
$$

\section{Method of Solution: Implementation of the HAM in Beam Vibrations}

For convenience of the reader, we present a brief description of the HAM in Appendix B.

Under the transformation $\tau=\omega t$ and $W(\tau)=u(t),(23)$ becomes as follows:

$$
\omega^{2} \ddot{W}+\alpha W+\beta W^{3}=0 .
$$

The zero-order deformation equation can be written as below

$$
(1-q) L\left[\varphi(\tau ; q)-W_{0}(\tau)\right]=q h \hbar(\tau) N[\varphi(\tau ; q)]
$$

in which

$$
N[\varphi(\tau ; q)]=\omega^{2} \frac{\partial^{2} \varphi(\tau ; q)}{\partial \tau^{2}}+\alpha \varphi(\tau ; q)+\beta \varphi(\tau ; q)^{3}=0 .
$$

We chose the following auxiliary linear operator as:

$$
L[\varphi(\tau ; q)]=\omega_{0}^{2}\left[\frac{\partial^{2} \varphi(\tau ; q)}{\partial \tau^{2}}+\varphi(\tau ; q)\right] .
$$

We employ Taylor expansion series for $\varphi(t ; q)$ and $\omega(q)$ as

$$
\begin{gathered}
\varphi(\tau ; q)=\varphi(\tau ; 0)+\left.\sum_{m=1}^{\infty} \frac{1}{m !} \frac{\partial^{m} \varphi(\tau ; q)}{\partial q^{m}}\right|_{q=0} \\
\times q^{m}=W_{0}(\tau)+\sum_{m=1}^{\infty} W_{m}(\tau) q^{m}, \\
\omega(q)=\omega_{0}+\left.\sum_{m=1}^{\infty} \frac{1}{m !} \frac{\partial^{m} \omega(q)}{\partial q^{m}}\right|_{q=0} q^{m}=\omega_{0}+\sum_{m=1}^{\infty} \omega_{m} q^{m} .
\end{gathered}
$$

In order to satisfy the initial conditions, the initial guess of $W(\tau)$ is chosen as follows:

$$
W_{0}(\tau)=W_{\max } \cos (\tau)
$$

In our case, to obtain the first-order approximation, the function of $W_{1}(\mathrm{t})$ can be expressed as (see Appendix B)

$$
\begin{gathered}
L\left[W_{1}(t)\right]=\left.\hbar h(t) N[\phi(t ; p)]\right|_{p=0}, \\
W_{1}(0)=0, \frac{d W_{1}(0)}{d t}=0 .
\end{gathered}
$$

Assuming $\hbar=-1, h(\mathrm{t})=1$ and after substituting (32) in (33), one would get

$$
\begin{gathered}
\omega_{0}^{2}\left(\ddot{W}_{1}+W_{1}\right)=W_{\max } \cos (\tau)\left(\omega_{0}^{2}-\alpha-\frac{3}{4} \beta W_{\max }^{2}\right) \\
-\frac{\beta W_{\max }{ }^{3}}{4} \cos (3 \tau), \\
W_{1}(0)=0, \quad \dot{W}_{1}(0)=0 .
\end{gathered}
$$

Eliminating the secular term, we have

$$
\omega_{0}=\sqrt{\alpha+\frac{3}{4} \beta W_{\max }^{2}} .
$$

Solving (35) and (36), the $W_{1}(\tau)$ is obtained as follows:

$$
W_{1}(\tau)=\frac{L_{1}}{8 \omega_{0}^{2}}(\cos (\tau)-\cos (3 \tau)),
$$

where

$$
L_{1}=-\frac{1}{4} \beta W_{\max }^{3} .
$$

Thus the first-order approximation of the $W(\tau)$ yields to,

$$
W(\tau)=W_{0}(\tau)+W_{1}(\tau)
$$

in which

$$
\tau=\omega t, \quad \omega=\omega_{0} .
$$

\section{Numerical Results and Discussion}

To validate the presented relations, the results of the natural frequency for the single isotropic lamina and the LCB with simple and clamped end conditions are compared with the available results in the literatures.

The variables used in the tables and plots are the nonlinear to linear frequency ratio, $\omega_{\mathrm{NL}} / \omega_{\mathrm{L}}$, the nondimensional amplitude of the vibration, $W_{\max }$, and the postbuckling load to critical load, $P_{\mathrm{NL}} / P_{\mathrm{cr}}$, (henceforth referred to as the frequency ratio, the amplitude ratio, and the buckling load ratio, resp.).

The materials steel and AS4/3501 Graphite-Epoxy, having the following mechanical properties, are used for the study.

Steel:

$$
\begin{gathered}
E=206.8 \mathrm{Gpa}, \quad G=79 \mathrm{Gpa}, \\
v=0.3, \quad \rho=7800 \mathrm{~kg} / \mathrm{m}^{3} .
\end{gathered}
$$

AS4/3501 graphite/epoxy:

$$
\begin{gathered}
E_{11}=144.8 \mathrm{Gpa}, \quad E_{22}=9.65 \mathrm{Gpa}, \\
G_{12}=4.14 \mathrm{Gpa}, \quad G_{13}=4.14 \mathrm{Gpa}, \\
G_{23}=4.14 \mathrm{Gpa}, \quad v_{12}=0.3, \quad \rho=1389.23 \mathrm{~kg} / \mathrm{m}^{3} .
\end{gathered}
$$


TABLE 1: Comparison of the frequency ratio of the isotropic single layer beam with simple end conditions for different amplitude ratio.

\begin{tabular}{|c|c|c|c|c|c|c|c|c|c|c|c|}
\hline \multirow[t]{3}{*}{$W_{\max }$} & \multicolumn{3}{|c|}{$\begin{array}{l}\text { For Slender beams without considering } \\
\text { shear deformation and rotary inertia }\end{array}$} & \multicolumn{8}{|c|}{$\eta=L / r$} \\
\hline & \multirow{2}{*}{ Ref. [1] } & \multirow{2}{*}{ Ref. [13] } & \multirow{2}{*}{ Ref. [14] } & \multicolumn{2}{|l|}{10} & \multicolumn{2}{|l|}{25} & \multicolumn{2}{|l|}{50} & \multicolumn{2}{|l|}{100} \\
\hline & & & & Ref. [15] & Present & Ref. [15] & Present & Ref. [15] & Present & Ref. [15] & Present \\
\hline 1 & 1.0892 & 1.0891 & 1.0897 & $1.11162(7)^{\mathrm{a}}$ & 1.1121 & $1.09253(5)$ & 1.0940 & $1.08971(5)$ & 1.0908 & $1.08900(5)$ & 1.0900 \\
\hline 2 & 1.3178 & 1.3177 & 1.3229 & $1.37006(16)$ & 1.3721 & $1.32130(8)$ & 1.3268 & $1.31425(7)$ & 1.3264 & $1.31249(7)$ & 1.3230 \\
\hline 3 & 1.6257 & 1.6256 & 1.6394 & $1.68839(47)$ & 1.6981 & $1.61464(14)$ & 1.6245 & $1.60520(11)$ & 1.6057 & $1.60295(11)$ & 1.6399 \\
\hline 4 & 1.9761 & - & - & - & 2.2190 & - & 2.0366 & - & 2.0092 & - & 2.0023 \\
\hline
\end{tabular}

${ }^{a}$ Numbers in the parentheses indicate the number of iterations required to achieve an accuracy of $10^{-6}$ in the evaluation of non-dimensional nonlinear frequency.

TABLE 2: Comparison of the frequency ratio of the isotropic single-layer beam with clamped end conditions for different amplitude ratio.

\begin{tabular}{lccccccc}
\hline \multirow{2}{*}{$\mathrm{W}_{\max }$} & \multicolumn{2}{c}{$\eta=25$} & \multicolumn{2}{c}{$\eta=50$} & \multicolumn{2}{c}{$\eta=100$} & \multicolumn{2}{c}{$\eta=500$} \\
& Ref. [17] & Present & Ref. [17] & Present & Ref. [17] & Present & Ref. [17] \\
\hline 1 & 1.0276 & 1.0299 & 1.0242 & 1.0260 & 1.0234 & 1.0240 & 1.0231 \\
2 & 1.1058 & 1.1068 & 1.0933 & 1.0987 & 1.0902 & 1.0910 & 1.0892 \\
3 & 1.2239 & 1.2302 & 1.1987 & 1.2131 & 1.1924 & 1.1950 & 1.1903 \\
4 & 1.3711 & 1.3856 & 1.3313 & 1.3421 & 1.3212 & 1.3288 & 1.3179 \\
\hline
\end{tabular}

TABLE 3: Comparison of the frequency ratio of the LCB with simple end conditions for different amplitude ratio $(\eta=34.6410)$.

\begin{tabular}{|c|c|c|c|c|c|c|}
\hline \multirow{2}{*}{$W_{\text {max }}$} & \multicolumn{2}{|c|}{ Cross-ply [0/90/90/0] } & \multicolumn{2}{|c|}{ Angle-ply $[45 /-45 /-45 / 45]$} & \multicolumn{2}{|c|}{ General lay-up [30/50/50/30] } \\
\hline & Ref. [7] & Present & Ref. [7] & Present & Ref. [7] & Present \\
\hline 5 & 1.0253 & 1.0308 & 1.0386 & 1.0397 & 1.0358 & 1.0422 \\
\hline 1 & 1.0975 & 1.1181 & 1.1466 & 1.1491 & 1.1365 & 1.1451 \\
\hline 2 & 1.3475 & 1.3543 & 1.5021 & 1.5536 & 1.4711 & 1.5471 \\
\hline 3 & 1.6822 & 1.7030 & 1.9541 & 1.9949 & 1.9004 & 2.0032 \\
\hline 4 & 2.1054 & 2.2064 & 2.4484 & 2.4807 & 2.3723 & 2.4020 \\
\hline
\end{tabular}

The shear correction factor $k$ is taken as $5 / 6$, as commonly used in the literature. All the layers are of equal thickness. Fiber orientation is measured from $x$-axis.

The validity of the results is established by Tables 1 and 3 for simple end conditions and Table 2 for clamped end conditions. Table 1 shows the frequency ratio for the single isotropic beam along with the results from literatures. It can be seen that the frequency obtained from two different beam models (i.e., Bernoulli-Euler [1, 13, 14] and Timoshenko [15] beams) yields the same value provided that the beam's slenderness ratio be at least 100 . This finding is also reported by [16] in which only the linear analysis was studied. The same results from [17] are included in Table 2 for the sake of comparison, and the agreement of the values of frequency ratio is good for various values of the amplitude and slenderness ratios.

Also, comparison of the frequency ratio for four-layer symmetric cross-ply, angle-ply and general lay-up composite beams with the finite element solutions from [7] for different amplitude ratio is reported in Table 3. As can be seen from these tables, the agreement between the results is quite good.
Figure 2 illustrates the effects of shear deformation and rotary inertia on the frequency ratio for three different amplitude ratios. It is clear that the CLT predicts the frequency ratio lower than the one obtained by FSDT. For thin beams and based on the two considered beam models, namely, CLT and FSDT, almost no difference is seen for different slenderness ratio.

The same layers, that is, layers oriented at $0,90,90$, and 0 , in two different configurations are considered in Figure 3 to examine the stacking sequence on the frequency ratio. As can be observed, the frequency ratio for [0/90/90/0] configuration is higher than the [90/0/0/90] configuration. Furthermore, as the amplitude ratio increases, the frequency ratio increases for two considered lamination schemes.

Figure 4 displays the frequency ratio versus the angle of orientation for the symmetric angle-ply $(\theta /-\theta /-\theta / \theta)$ thin and moderately thick LCB and for three different amplitude ratios and two considered boundary conditions. It is evident that all figures for thin and thick beams reach their maximum values at the orientation around $\left(15^{\circ}-20^{\circ}\right)$. This result is also reported by [18] in which only the linear study was conducted. In the intermediate region $\left(60^{\circ} \leq \theta \leq 90^{\circ}\right)$, the frequency ratio remains almost unchanged. 


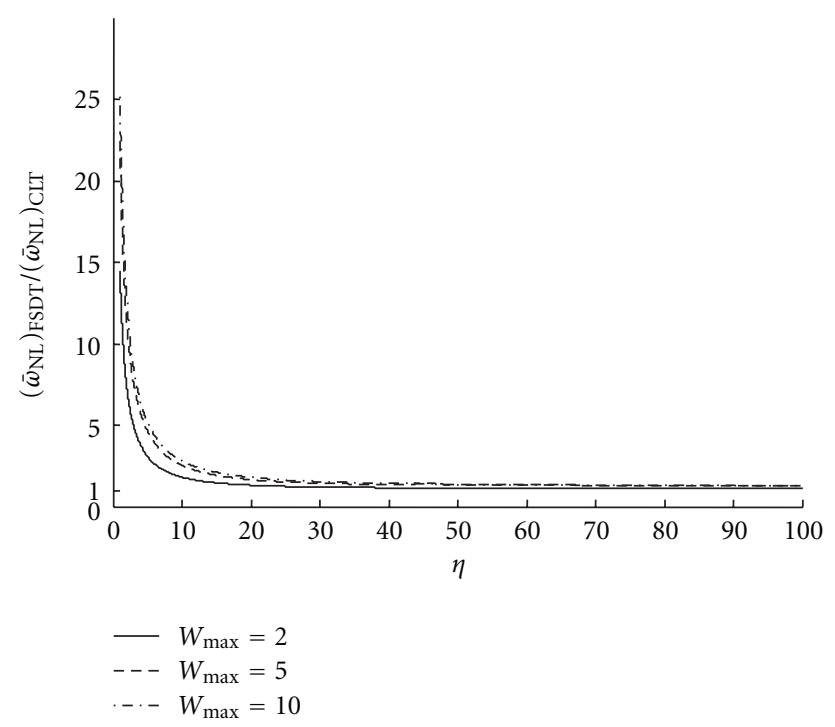

FIGURE 2: Effects of shear deformation and rotary inertia on the frequency ratio versus slenderness ratio for the [0/90/90/0] configuration.

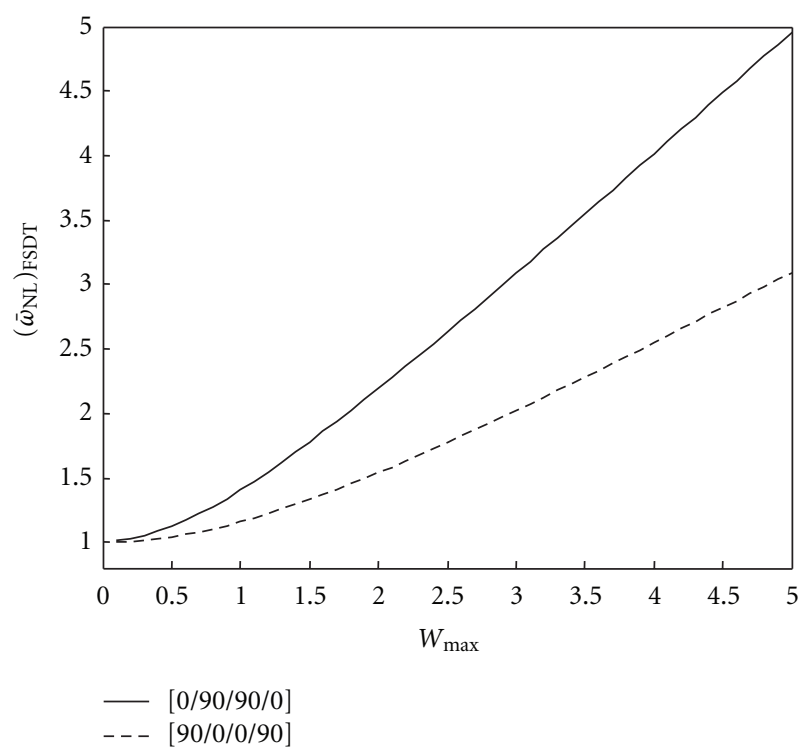

FIGURE 3: Effect of ply orientation on the frequency ratio of the LCB for $\eta=10$.

Variation of the buckling load ratio versus amplitude ratio is shown in Figure 5. As can be observed for thick beams, there are significant differences between buckling load ratio predicted by CLT and FSDT. This difference is more considerable at larger amplitudes.

\section{Conclusions}

Analytical expressions for the frequency and deflection equations of the LCB at large amplitude on the basis of FSDT with simple end conditions have been derived. The applicability of the theory is demonstrated by numerical results, which show good agreement with published results.

Limited numerical studies are conducted to examine the effect of slenderness ratio, fiber orientation, and amplitude of vibration on the vibration and buckling characteristics of the LCB. The present study can serve as a quick and accurate reference to predict the vibration and postbuckling response of the composite beam at any amplitude.

\section{Appendices}

\section{A. Derivation of Bending (EI), Torsional (GJ), Bending-Torsion Coupling (C), and Shear (kAG) Rigidities}

Based on CLT, the constitutive equations of the laminate relating the stress resultant and the curvatures can be written as

$$
\left\{\begin{array}{l}
M_{x} \\
M_{y} \\
M_{x y}
\end{array}\right\}=\left[\begin{array}{lll}
D_{11} & D_{12} & D_{16} \\
D_{12} & D_{22} & D_{26} \\
D_{16} & D_{26} & D_{66}
\end{array}\right]\left\{\begin{array}{c}
k_{x} \\
k_{y} \\
k_{x y}
\end{array}\right\},
$$

where $D_{i j}$ are anisotropic stiffness coefficients which are defined by

$$
D_{i j}=\int_{-t / 2}^{t / 2} \bar{Q}_{i j} z^{2} d z, \quad(i, j=1,2,6)
$$

where $\bar{Q}_{i j}$ and $t$ are the transformed material constants [19] and thickness of the beam. The moment $M_{y}$ can be considered to be zero. Then $k_{y}$ can be eliminated from (A.1) to give

$$
\left\{\begin{array}{c}
M_{x} \\
M_{x y}
\end{array}\right\}=\left[\begin{array}{cc}
D_{11}-\frac{D_{12}^{2}}{D_{22}} & D_{16}-\frac{D_{12} D_{26}}{D_{22}} \\
D_{16}-\frac{D_{12} D_{26}}{D_{22}} & D_{66}-\frac{D_{26}^{2}}{D_{22}}
\end{array}\right]\left\{\begin{array}{c}
k_{x} \\
k_{x y}
\end{array}\right\} .
$$

The bending $M_{x}$ and twisting $M_{x y}$ moments can be related to the resultant bending $(M)$ and torsional $(T)$ moments as follows [20]:

$$
M=-b M_{x}, \quad T=2 b M_{x y} .
$$

Also, the curvatures $k_{x}$ and $k_{x y}$ can be related to the bending slope $\theta^{\prime}$ and twist rate $\psi^{\prime}$ so that [20]

$$
k_{x}=-\theta^{\prime}, \quad k_{x y}=2 \psi^{\prime} .
$$

Thus, the expressions for $M$ and $T$ are given by

$$
\left\{\begin{array}{c}
M \\
T
\end{array}\right\}=b\left[\begin{array}{cc}
D_{11}-\frac{D_{12}{ }^{2}}{D_{22}} & 2\left(D_{16}-\frac{D_{12} D_{26}}{D_{22}}\right) \\
2\left(D_{16}-\frac{D_{12} D_{26}}{D_{22}}\right) & 4\left(D_{66}-\frac{D_{26}{ }^{2}}{D_{22}}\right)
\end{array}\right]\left\{\begin{array}{l}
\theta^{\prime} \\
\psi^{\prime}
\end{array}\right\} .
$$



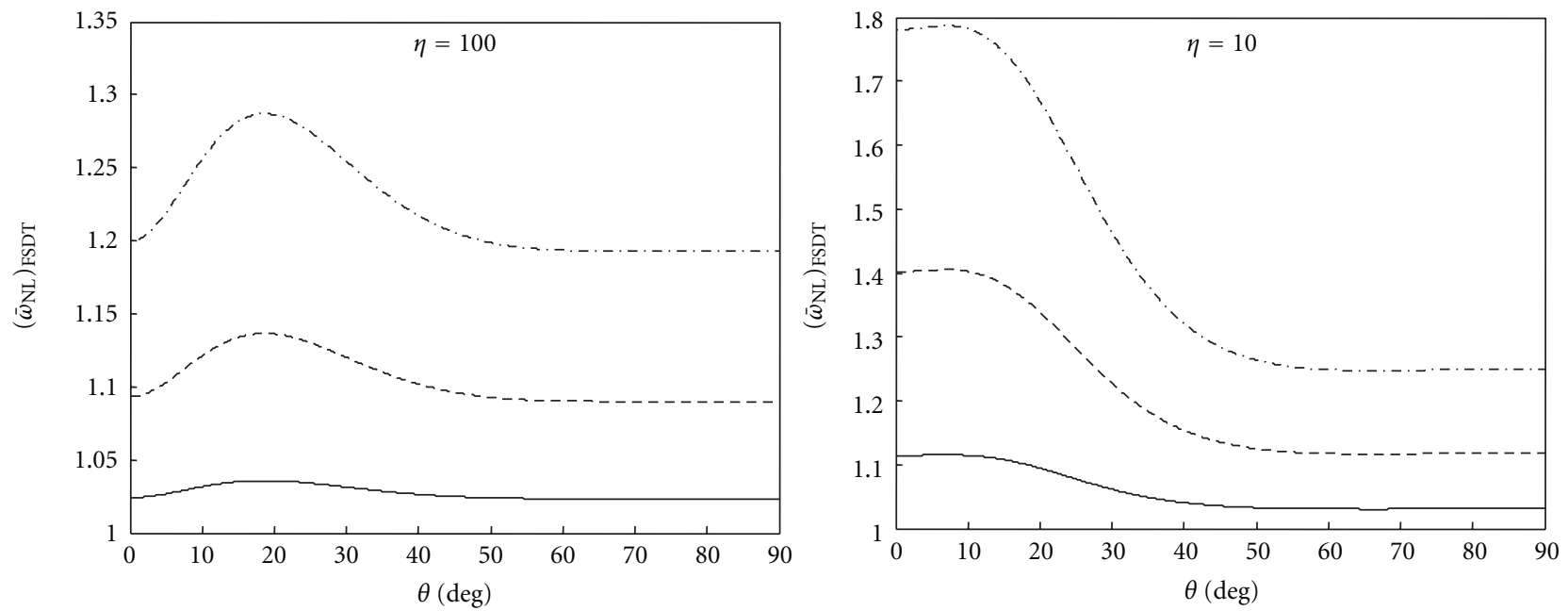

(a) Simple End Condition
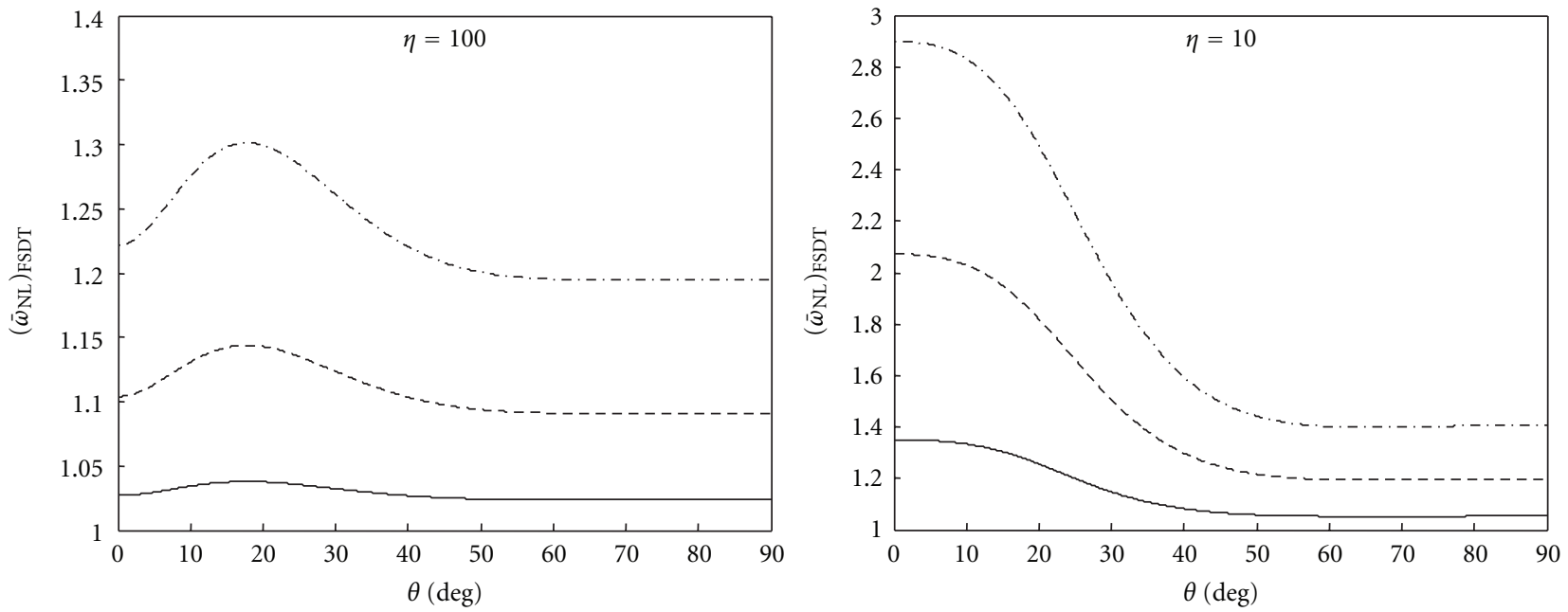

(b) Clamped End Condition

FIgURE 4: Variation of the frequency ratio versus the angle of orientation for the angle-ply $\operatorname{LCB}[\theta,-\theta,-\theta, \theta]$ at two slenderness ratios. $\left(-: w_{\max }=1,--: w_{\max }=2,-\cdot-: w_{\max }=5\right)$.

The common relationship between the bending $M$ and torsional $T$ moments with the bending slope $\theta^{\prime}$ and twist rate $\psi^{\prime}$ are as follows:

$$
\left\{\begin{array}{c}
M \\
T
\end{array}\right\}=\left[\begin{array}{cc}
\text { EI } & C \\
C & G J
\end{array}\right]\left\{\begin{array}{c}
\theta^{\prime} \\
\psi^{\prime}
\end{array}\right\}
$$

An element-by-element comparison of (A.6) and (A.7), we obtain

$$
\begin{gathered}
\mathrm{EI}=b\left(D_{11}-\frac{D_{12}{ }^{2}}{D_{22}}\right), \quad C=2 b\left(D_{16}-\frac{D_{12} D_{26}}{D_{22}}\right), \\
\mathrm{GJ}=4 b\left(D_{66}-\frac{D_{26}{ }^{2}}{D_{22}}\right) .
\end{gathered}
$$

The transverse shear force-strain relation for the LCB can be expressed as

$$
Q_{x z}=\mathrm{kb}\left(\int_{-t / 2}^{t / 2} \bar{Q}_{55} d \hat{z}\right) \gamma_{x z}=\mathrm{kAG} \gamma_{x z}
$$

where:

$$
\mathrm{kAG}=\mathrm{kb} \int_{-t / 2}^{t / 2} \bar{Q}_{55} d \widehat{z} .
$$

\section{B. An Overview of Homotopy Analysis Method (HAM)}

For convenience of the reader, we will first present a brief description of the HAM [21]. Consider the following nonlinear homogeneous differential equations:

$$
N[u(t)]=0,
$$




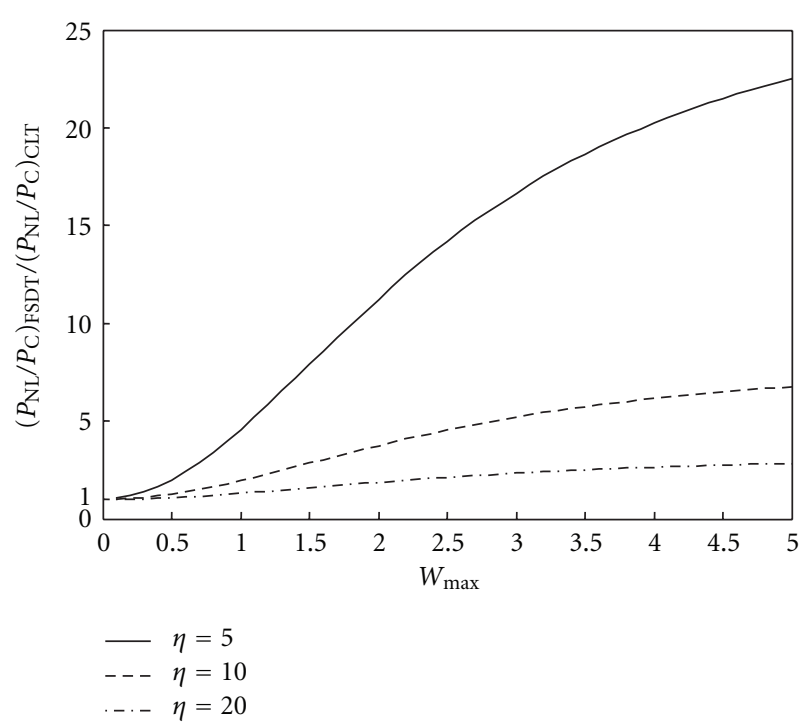

FIGURE 5: Effects of shear deformation and rotary inertia on the buckling load ratio versus amplitude ratio for the [0/90/90/0] configuration.

where $N$ is nonlinear operators, $t$ denotes the independent variable, and $u(t)=0$ are unknown functions.

Liao constructed the so-called zero-order deformation equation as [21]

$$
(1-p) L\left[\phi(t ; p)-u_{0}(t)\right]=p \hbar h(t) N[\phi(t ; p)],
$$

where $p \in[0,1]$ is an embedding parameter, $\hbar$ are nonzero auxiliary functions, $h(\mathrm{t})$ are nonzero auxiliary function, $L$ is an auxiliary linear operator, $u_{0}(t)$ are initial guesses of $u(t)$, and $\phi(\mathrm{t} ; \mathrm{p})$ are unknown functions. As $p$ increases from 0 to 1 , the $\phi(t ; p)$ varies from the initial approximation to the exact solution. In other words, $\phi(t ; 0)=u_{0}(t)$ and $\phi(t ; 1)=$ $u(t)$.

The deformation equation (B.2) can provide us with a family of approximation series whose convergence region depends upon the auxiliary parameter $\hbar$ and the auxiliary function $h(\mathrm{t})$. More importantly, this provides us with a simple way to adjust and control the convergence regions and rates of approximation series.

Differentiating once more from (B.2) with respect to the embedding parameter $p$ and then setting $p=0$, we obtain the first-order deformation equation as

$$
L\left[u_{1}\right]=\hbar h(t) N[\phi(t ; p)]_{p=0}
$$

which gives the first-order approximation for the $u(t)$ [21]. The higher order approximation of the solution can be obtained by calculating the $m$-order $(m>1)$ deformation equation which can be calculated by differentiating (B.3) $m$ times with respect to the $p$ and then setting $p=0$ and finally dividing them by $m$ ! [21]. Therefore, the $m$ th-order deformation equation can be obtained as follows:

$$
L\left[u_{m}(t)-u_{m-1}(t)\right]=\hbar h(t) R_{m}\left(\vec{u}_{m-1}, \ldots, \vec{u}_{m-1}\right),
$$

where $\left(\vec{u}_{m-1}, \ldots, \vec{u}_{m-1}\right)$ and $R_{m}\left(\vec{u}_{m-1}, \ldots, \vec{u}_{m-1}\right)$ are defined as

$$
\begin{array}{r}
R_{m}\left(\vec{u}_{m-1}, \ldots, \vec{u}_{m-1}\right)=\left.\frac{1}{(m-1) !} \frac{d^{m-1} N[\phi(t ; p)]}{d p^{m-1}}\right|_{q=0}, \\
\vec{u}_{m-1}=\left\{u_{0}, u_{1}, u_{2}, \ldots, u_{m-1}\right\}
\end{array}
$$

subjected to the following initial conditions:

$$
u_{m}(0)=0, \quad \dot{u}_{m}(0)=0 .
$$

To solve (B.2), we employ Taylor expansion series for $\phi(t ; p)$ as

$$
\begin{aligned}
\phi(t ; p) & =\phi(t ; 0)+\left.\sum_{m=1}^{\infty} \frac{1}{m !}\left(\frac{\partial^{m} \phi(t ; p)}{\partial p^{m}}\right)\right|_{p=0} p^{m} \Longrightarrow u(t) \\
& =u_{0}(t)+\sum_{m=1}^{\infty} u_{m}(t) p^{m},
\end{aligned}
$$

where $u_{m}(t)$ is called the $m$ th-order derivative of unknown function $\phi(t ; p)$.

\section{Acknowledgment}

The authors are grateful for the encouragement and useful comments of the reviewers.

\section{References}

[1] M. Baghani, R. A. Jafari-Talookolaei, and H. Salarieh, "Large amplitudes free vibrations and post-buckling analysis of unsymmetrically laminated composite beams on nonlinear elastic foundation," Applied Mathematical Modelling, vol. 35, no. 1, pp. 130-138, 2011.

[2] P. Malekzadeh and A. R. Vosoughi, "DQM large amplitude vibration of composite beams on nonlinear elastic foundations with restrained edges," Communications in Nonlinear Science and Numerical Simulation, vol. 14, no. 3, pp. 906-915, 2009.

[3] B. P. Patel, M. Ganapathi, and M. Touratier, "Nonlinear free flexural vibrations/post-buckling analysis of laminated orthotropic beams/columns on a two parameter elastic foundation," Composite Structures, vol. 46, no. 2, pp. 189-196, 1999.

[4] S. A. Emam and A. H. Nayfeh, "Postbuckling and free vibrations of composite beams," Composite Structures, vol. 88, no. 4, pp. 636-642, 2009.

[5] R. K. Kapania and S. Raciti, "Nonlinear vibrations of unsymmetrically laminated beams," AIAA Journal, vol. 27, no. 2, pp. 201-210, 1989.

[6] M. Ganapathi, B. P. Patel, J. Saravanan, and M. Touratier, "Application of spline element for large-amplitude free vibrations ," Composites Part B, vol. 29B, no. 1, pp. 1-8, 1998.

[7] M. Kiran, I. Bangera, and K. Chandrashekhara, "Nonlinear vibration of moderately thick laminated beams using finite element method," Finite Elements in Analysis and Design, vol. 9, no. 4, pp. 321-333, 1991. 
[8] A. W. Obst and R. K. Kapania, "Nonlinear static and transient finite element analysis of laminated beams," Composites Engineering, vol. 2, no. 5-7, pp. 375-389, 1992.

[9] J. R. Banerjee and F. W. Williams, "Exact dynamic stiffness matrix for composite timoshenko beams with applications," Journal of Sound and Vibration, vol. 194, no. 4, pp. 573-585, 1996.

[10] J. R. Banerjee, "Free vibration of axially loaded composite Timoshenko beams using the dynamic stiffness matrix method," Computers and Structures, vol. 69, no. 2, pp. 197208, 1998.

[11] G. V. Rao, K. M. Saheb, and G. R. Janardhan, "Fundamental frequency for large amplitude vibrations of uniform Timoshenko beams with central point concentrated mass using coupled displacement field method," Journal of Sound and Vibration, vol. 298, no. 1-2, pp. 221-232, 2006.

[12] S. Woinowsky-Krieger, "The effect of an axial force on the vibration of hinged bars," Journal of Applied Mechanics, vol. 17, pp. 35-36, 1950.

[13] L. Azrar, R. Benamar, and R. G. White, "A semi-analytical approach to the non-linear dynamic response problem of S$\mathrm{S}$ and C-C beams at large vibration amplitudes part I: general theory and application to the single mode approach to free and forced vibration analysis," Journal of Sound and Vibration, vol. 224, no. 2, pp. 183-207, 1999.

[14] M. I. Qaisi, "Application of the harmonic balance principle to the nonlinear free vibration of beams," Applied Acoustics, vol. 40, no. 2, pp. 141-151, 1993.

[15] G. V. Rao, I. S. Raju, and K. K. Raju, "Nonlinear vibrations of beams considering shear deformation and rotary inertia," AIAA Journal, vol. 14, no. 5, pp. 685-687, 1976.

[16] S. M. Han, H. Benaroya, and T. Wei, "Dynamics of transversely vibrating beams using four engineering theories," Journal of Sound and Vibration, vol. 225, no. 5, pp. 935-988, 1999.

[17] G. V. Rao and K. K. Raju, "A numerical integration method to study the large amplitude vibration of slender beams with immovable ends," Journal of the Institution of Engineers, vol. 83 , pp. 42-44, 2002.

[18] E. Chandrasekaran, K. Jayaraman, and S. M. Nazeer, "Effects of symmetric and antisymmetric fiber orientations on the natural frequencies of FRP aircraft panel boards," Journal of Reinforced Plastics and Composites, vol. 23, no. 8, pp. 831-841, 2004.

[19] R. M. Jones, Mechanics of Composite Materials, McGraw-Hill, NewYork, NY, USA, 1975.

[20] T. A. Weisshaar and B. L. Foist, "Vibration tailoring of advanced composite lifting surfaces," Journal of Aircraft, vol. 22, no. 2, pp. 141-147, 1985.

[21] S. Liao, Beyond Perturbation-Introduction to the Homotopy Analysis Method, Chapman \& Hall/CRC, 2004. 

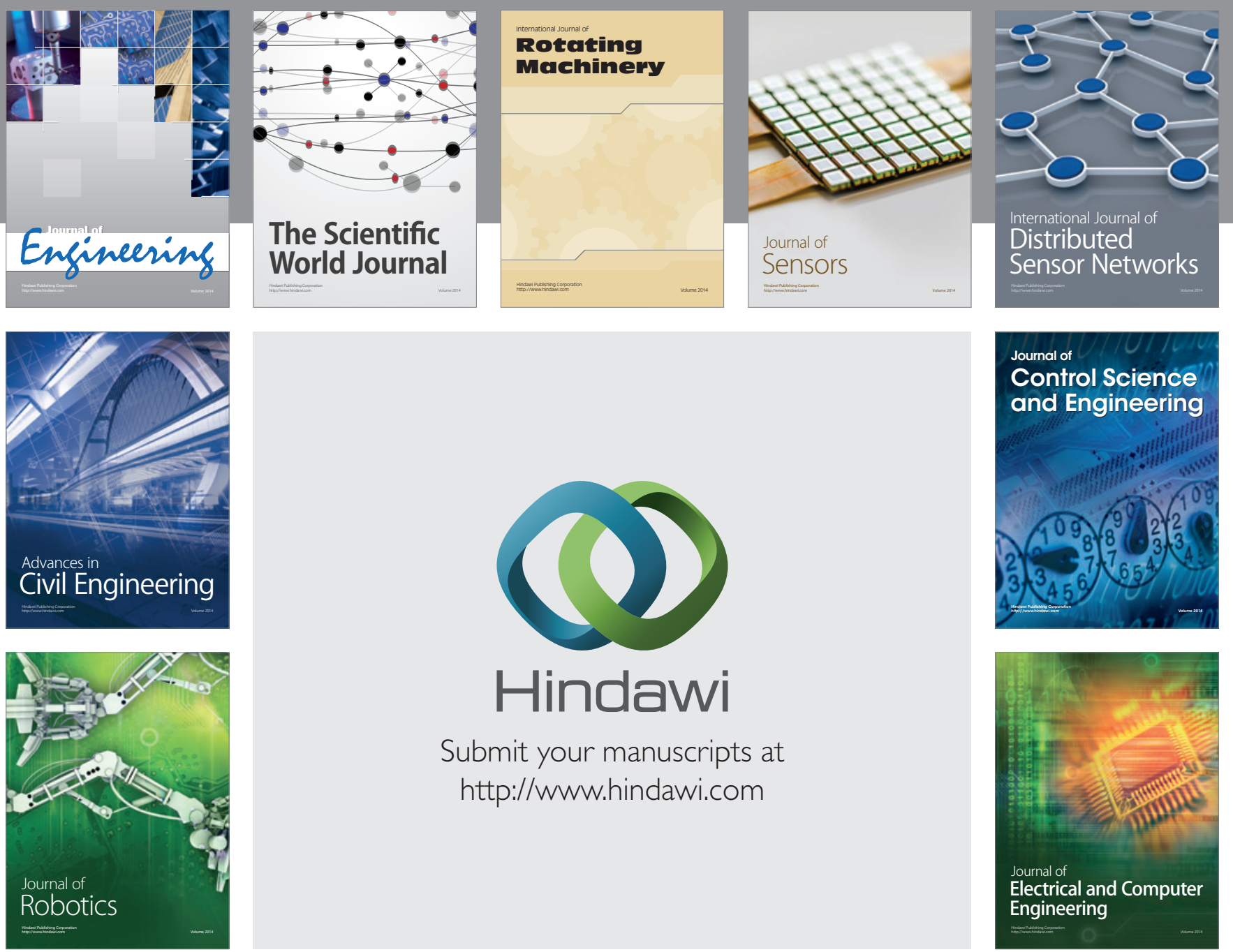

Submit your manuscripts at

http://www.hindawi.com
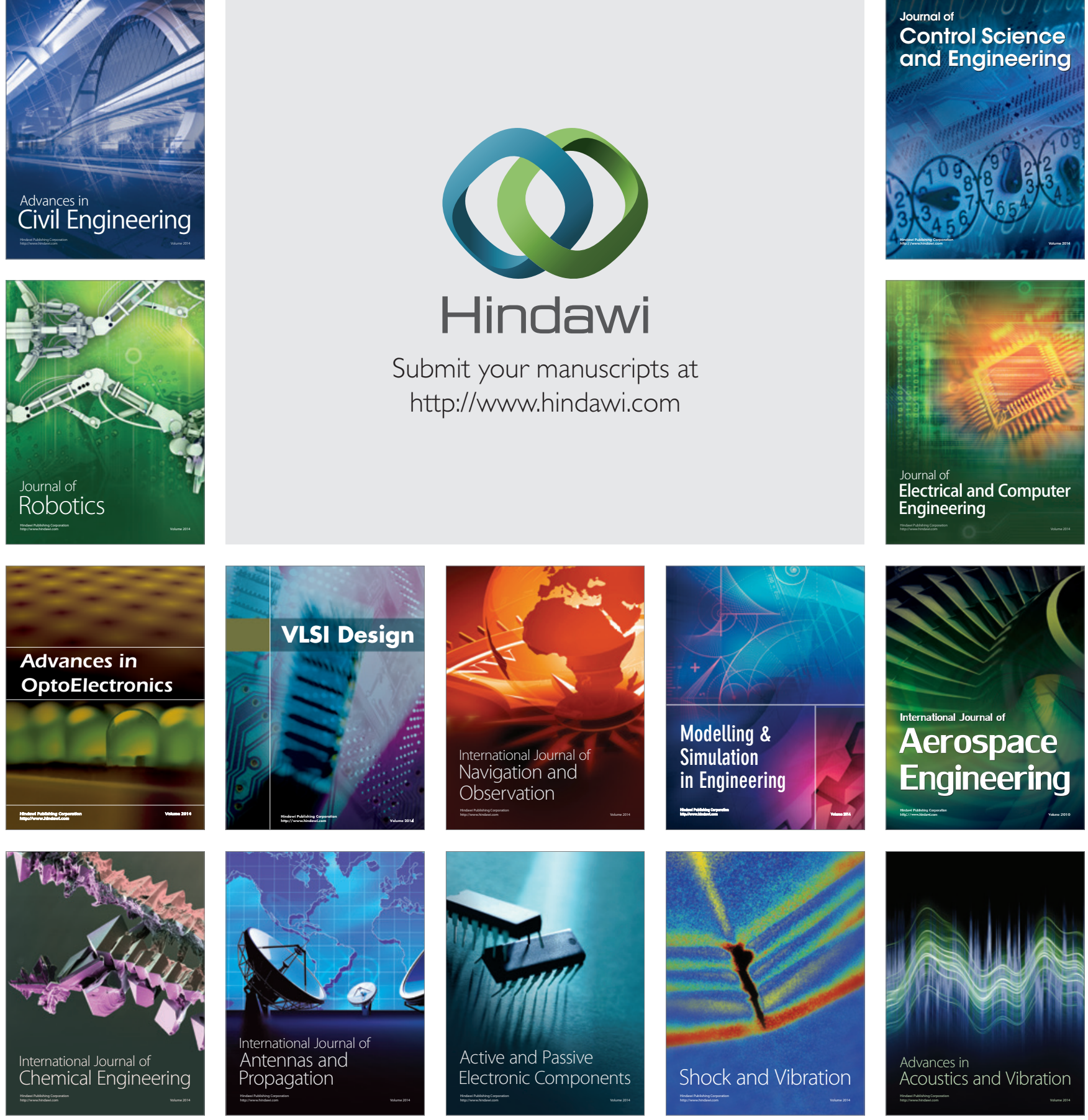